\title{
Classification of hexagonal adlayer arrangements by means of collective geometrical properties
}

\author{
Alexandre Tkatchenko and Nikola Batina ${ }^{a)}$ \\ Departamento de Química, División de Ciencias Básicas e Ingeniería, \\ Universidad Autónoma Metropolitana-Iztapalapa, Avenida San Rafael Atlixco 186, Vicentina, \\ AP 55-534, México, Distrito Federal 09340, Mexico
}

(Received 28 July 2006; accepted 13 September 2006; published online 24 October 2006)

Unequal-sphere packing model is applied for the simulation of large number of hexagonal adlayer structures with surface coverage between $\theta=\frac{1}{3}$ and $\theta=1$ on the hexagonal substrate, with atomic radius of the adsorbate and substrate atoms as the only input. Each structure is characterized with respect to collective adlayer properties: the average adlayer height and the adlayer roughness. The distribution of hexagonal arrangements is presented in a special plot, which can be used for identification and characterization of hexagonal adlayers of different surface coverages and atomic registries. The most likely structures are related to the extreme values of our model parameters. The usefulness of this methodology is successfully demonstrated by comparison with some real adsorbate-substrate systems, i.e., halogens and rare gases adsorbed on (111) surface. Besides the agreement with experimental results, our model offers new insight into the formation of atomic adlayers and detailed analysis of the atomic registry. We believe that our approach will be of use for identification of probable structures among the large number of combinatorial possibilities in theoretical studies and for better interpretation of experimental results (i.e., scanning-tunneling microscopy images of atomic adlayers). (C) 2006 American Institute of Physics.

[DOI: $10.1063 / 1.2360530]$

\section{INTRODUCTION}

The arrangement of adsorbates on well-defined substrates has been the subject of numerous different studies in surface and material sciences and modern electrochemistry. ${ }^{1-3}$ Results of these studies help us to understand the mechanisms of catalyst action, the electrical double layer structure, and adsorption phenomena. ${ }^{1-3}$ Indeed, some of the adsorbate-substrate systems have been successfully characterized at the atomic level by quantum mechanical methods, such as density functional theory ${ }^{4}$ (DFT) or Monte Carlo (MC) methods. ${ }^{5}$ Recent and rapid progress in nanoscience indicates that this knowledge could also be useful for future technological development. ${ }^{6,7}$ Still, such advancement is prevented by the lack of general knowledge related to the possibility of formation of different adsorbate arrangements at different substrates and by the fact that no methodology is available for selection of probable structures among all the combinatorial possibilities for an adsorbate-substrate system. Namely, the number of atoms involved in the formation of an adlayer is usually very large, thus making the traditionally used techniques intractable. Herein we focus on the development of an approach, which will allow an analysis of a large number of possibilities for the structure of atomic adlayer and select the most likely structures with only a few input parameters. We believe that development of simple models, such as the one proposed in this study, could allow detailed understanding regarding the atomic structure and formation of adlayers on well-ordered surfaces.

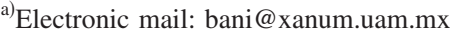

In this study, we focus on one particular case frequently reported in experimental studies, which involves formation of hexagonally ordered adlayers on face centered cubic (111) substrates. From a methodological point of view, we pursue several goals: determination of the "total" number of different hexagonal adlayer structures, identification of structural parameters which will lead to easy classification of the many hexagonal adlayer arrangements and comparison with real structures obtained in experiments. We use unequal-sphere packing (USP) model, ${ }^{8}$ which is based on packing of layers of spheres having a specific radius. The determination of stable structures is done according to the analysis of collective adlayer properties: the average adlayer height and the adlayer roughness. Selection and description of these two particular parameters were presented in our previous work, see Refs. 8 and 9. Our model, based on sphere packing, was developed for specific adsorbate-substrate system characterization. However, the packing of geometrical objects has been of interest and widely studied in physical sciences for description of complex materials before. ${ }^{10}$ For example, it has been recently shown that ellipsoidal particles can pack better in three dimensions (3D) than spherical particles, a quiet unexpected result. ${ }^{11,12}$ Therefore, we believe that models based on geometrical principles can still lead to many important discoveries.

\section{METHODOLOGY}

The height of each adsorbate particle (sphere) $(Z)$ is calculated in the surface-normal direction: 


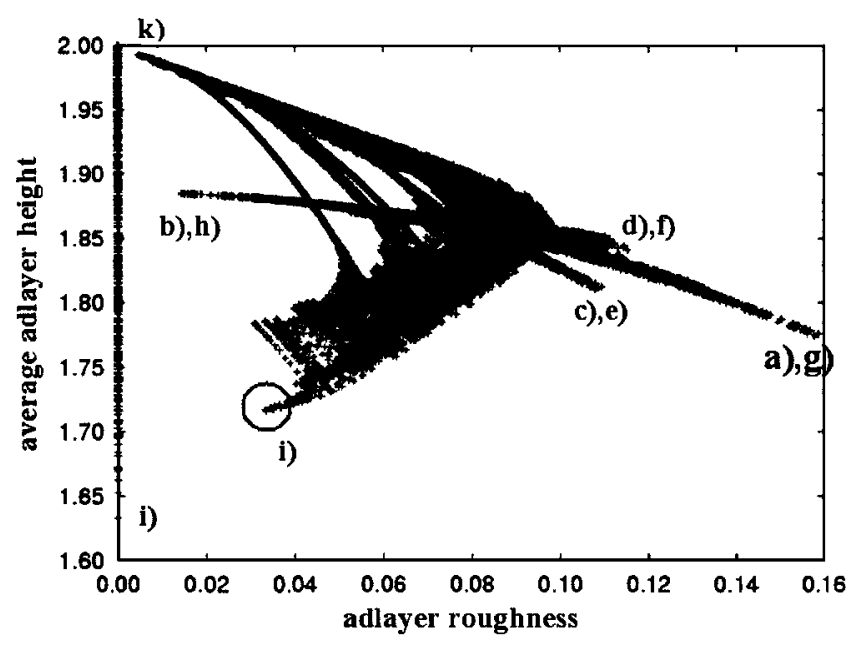

FIG. 1. Plot of average adlayer height vs adlayer roughness for a variety of hexagonal structures simulated in our study. Each point of the plot corresponds to specific hexagonal structure. The labels of the marked points refer to structures from Fig. 2.

$$
Z=\sqrt{\left(r_{1}+r_{2}\right)^{2}-\left(x-x_{s}\right)^{2}-\left(y-y_{s}\right)^{2}},
$$

where $r_{1}$ and $r_{2}$ are the substrate and adsorbate radii, respectively, $(x, y)$ is the adsorbate particle position, and $\left(x_{s}, y_{s}\right)$ is the closest substrate particle position.

The adlayer $(P)$ average height $[A(P)]$ and adlayer roughness $[R(P)]$ are calculated as follows:

$$
\begin{aligned}
& A(P)=\sum_{i=1}^{N} Z\left(P_{i}\right) / N, \\
& R(P)=\sqrt{\sum_{i=1}^{N}\left(Z\left(P_{i}\right)-A(P)\right)^{2} / N .}
\end{aligned}
$$

The adlayer and the substrate are arranged in rigid hexagonal order. The radii of adsorbate and substrate were taken to be equal to 1 . In case of larger adsorbate radius, no changes in simulation results were noticed. The surface coverage was gradually decreased from $\theta=1$ to $\theta=\frac{1}{3}$, which is achieved by increasing the interadsorbate distance in steps of 0.007 . For each interadsorbate distance, the adlayer rotation angle was changed from $0^{\circ}$ to $30^{\circ}$, in increments of $0.3^{\circ}$. Afterwards, the adlayer was allowed to shift laterally in the substrate unit cell with steps of 0.05 in the $X$ and 0.0577 in the $Y$ directions. For each interadsorbate distance, the simulation square size was adjusted in order to keep the adsorbate particle number constant (around 2000). A total of 24000000 hexagonal structures were analyzed. This simulation, equivalent to $750 \mathrm{~h}$ on a single $3 \mathrm{GHz}$ processor, was performed by means of parallel processing. Each hexagonal structure was characterized by two parameters: the average adlayer height and adlayer roughness, specifically introduced in our study.

\section{RESULTS AND DISCUSSION}

The plot in Fig. 1 represents the distribution of hexagonally ordered adlayers with respect to our model parameters. The shape of this graph is very interesting and irregular, with the majority of structures (points in the graph) in the middle of the plot. Clearly, the distribution of hexagonal structures is limited to a certain confined space. For example, a hexagonal adlayer with an average height of 1.7 and roughness equal to 0.16 does not exist. Few structures are found in the top-left corner with average adlayer height close to 2 (atop position of the adsorbate layer with very low value of the adlayer roughness) or structures with maximum adlayer roughness equal to 0.16 (right part of the graph) with modest distance from the substrate (1.78).

The origin of variations in the average adlayer height and adlayer roughness between hexagonal structures is due to differences in the adlayer atomic registry. Detailed analysis of this relation was done for certain structures marked as (a)-(k). The chosen structures were identified as extreme points in Fig. 1. The point marked with a circle was found to be a feature of our finite size simulation and was identified as a slightly rotated (i) structure. Although Fig. 1 does not include the surface coverage data explicitly, the chosen structures are ordered by increase in the surface coverage from (a) to $(\mathrm{k})$. Detailed analysis of the atomic registry for these structures is presented in Fig. 2. By combining information from Figs. 1 and 2 just for these selected extremes, we find that the structure $(\mathrm{a})$, characterized as $(2 \times 2) R 30^{\circ}$-symmetry, possesses the same atomic registry as structure $(\mathrm{g})$ $\left[(\sqrt{7} \times \sqrt{7}) R 19.1^{\circ}\right.$-symmetry]. Therefore in Fig. 1, they are found at the same position. The same conclusion could be drawn for other pairs of structures: (b) and (h), (d) and (f), and (c) and (e). This shows that hexagonally ordered adlayers with different surface coverage could occupy the same atomic registry. For example, (b) and (h) are always flat with all atoms positioned at the same adsorption site. Another interesting fact is the distribution of a particular arrangement $(\sqrt{7} \times \sqrt{7}) R 19.1^{\circ}$, which occupies space between two extreme points, denoted as $(\mathrm{g})$ and $(\mathrm{h})$ having maximum adlayer roughness or completely flat, respectively. Probably, the most understandable and the most simple is the pathway for arrangements with $(1 \times 1)$ and $(\sqrt{3} \times \sqrt{3}) R 30^{\circ}$ symmetries, with identical adlayer registry. Moving these adlayers over the substrate induces changes in the average adlayer height, but not in the adlayer roughness. Both arrangements are always flat and with atomic registry between threefold [structure (i)] and atop [structure (k)]. Results obtained from such kind of analysis could be useful in the search for structures with very specific properties, i.e., a certain distance from the substrate or adlayer roughness, which could allow the design of atomic assembly devices within the adsorbatesubstrate systems or be helpful for the analysis of experimental data.

In order to relate the observed structures with surface coverage, we plot the average adlayer height versus adlayer interadsorbate distance (Fig. 3). For each interadsorbate distance, we plot the average heights of minimum (solid line) and maximum (dashed line) positioned adlayer structures [see analogy of structures (i) and (k) with Fig. 1]. In Fig. 3, one can easily identify several minimum and maximum distinguished points, which we believe are related to the formation of the most probable structures. Note that all of these extreme points correspond to commensurate structures. 

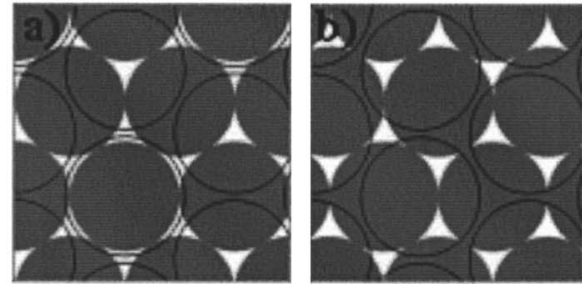

$\left.(2 \times 2) R 30^{\circ}-\pi\right)$
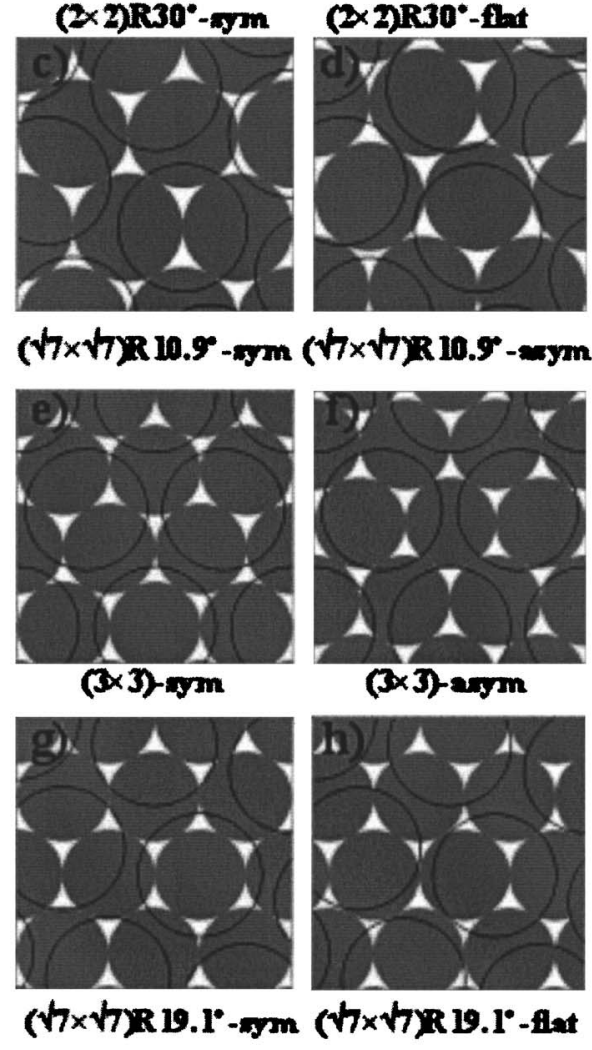

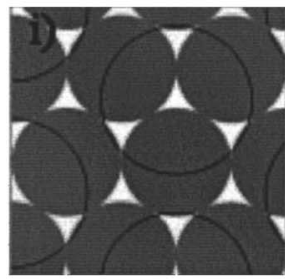

$(\sqrt{3} \times \sqrt{3}) \mathbf{R} 30^{*}-3$ fall

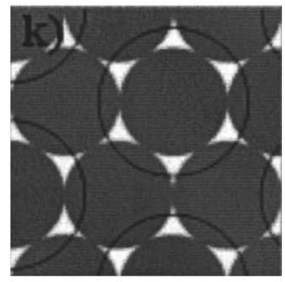

$(\sqrt{3} \times \sqrt{3}) \times 30^{\circ}-2 \operatorname{stop}$
FIG. 2. Transparent model of specific hexagonal structures, marked in Fig. 1. Large circles represent adsorbate, while smaller circles represent substrate. Note the same atomic registry between structures: (a) and (g), (b) and (h), (c) and (e), and (d) and (f).

In order to compare results of our simulation with real experiments and validate our approach, we use two sets of data obtained for adsorption of halogens (or halides) on (111) metal surfaces (chemisorption) and another set involving rare-gas adsorption on (111) fcc metal surfaces (physisorption). Both systems have been thoroughly investigated by different experimental techniques and are well known. For recent reviews of both systems, see Refs. 2 and 13. There is plenty of evidence of hexagonally ordered adlayers related to these two cases, many of which are commensurate with the substrate surface.

As the first attempt to correlate results of our simulation and real experimental data, we take the I-Pt(111) system. It

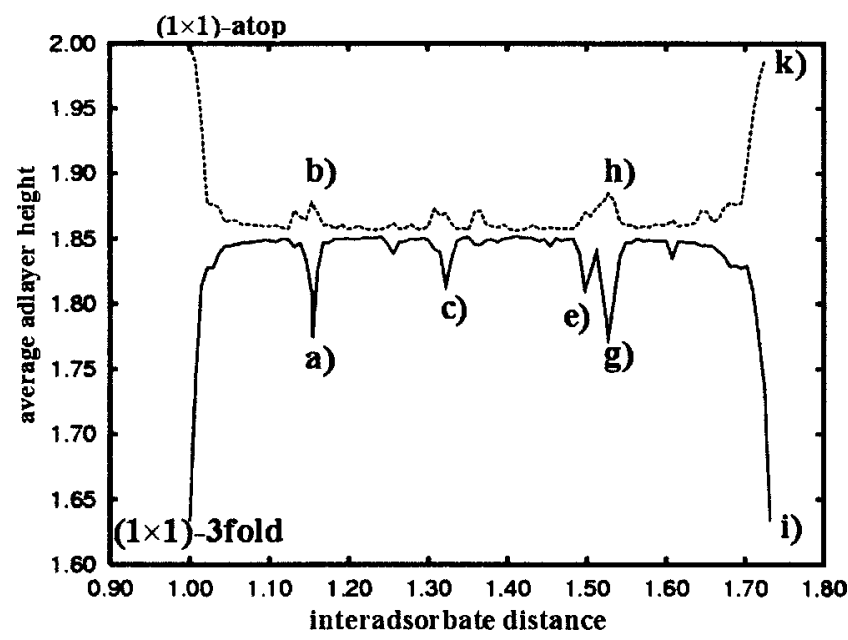

FIG. 3. Plot of average adlayer height vs interadsorbate distance for the surface coverage interval from $\theta=1$ to $\theta=\frac{1}{3}$. The labels refer to structures from Fig. 2.

is very well known that it forms structures (i), (g), and (e),,$^{2,3}$ which are clearly identified as minima in Fig. 3. See Ref. 8 for more detailed analysis of this case. Bromine on $\mathrm{Pt}(111)$ also forms a $(3 \times 3)$ [structure $(\mathrm{e})]$ and high-order commensurate structures, ${ }^{14}$ which we believe could be identified as intermediate minima between peaks (c) and (e). In general, in the case of halogen adsorption, it is established that the most stable adlayer structures are those which occupy threefold sites. ${ }^{2,3}$ According to our study, we could broaden this conclusion to structures with the minimum average adlayer height (the closest to substrate) as being the most likely ones. See the minimum adlayer height structures marked on Fig. 3. This example shows how our approach alone with available experimental data could identify important parameters for finding most likely structures from a large pool of possibilities for an adsorbate-substrate system.

In the case of the rare-gas adsorption, according to the most recent studies, ${ }^{13,15}$ i.e., as found for $\mathrm{Xe}-\mathrm{Pd}(111)$, the $(\sqrt{3} \times \sqrt{3}) R 30^{\circ}$-atop structure is formed, which is marked as (k) in Fig. 3. In addition, $(\sqrt{7} \times \sqrt{7}) R 19.1^{\circ}$ and $(\sqrt{19} \times \sqrt{19}) R 23.4^{\circ}$ structures were found for the same system by_different authors. ${ }^{16-18}$ Vogt et al. ${ }^{17}$ proposed that $(\sqrt{7} \times \sqrt{7}) R 19.1^{\circ}$ is arranged in a symmetric structure with atop and threefold adsorption sites, like the one presented in (g). In the same study, the threefold adsorption site was proposed for $(\sqrt{3} \times \sqrt{3}) R 30^{\circ}$ structure, marked as (i) in our graph. This is in contradiction with recent experimental ${ }^{13}$ and theoretical $^{15}$ findings, which postulate that the atop site is preferential for the rare-gas adsorption on metal surfaces instead of the threefold one.

In order to address this issue, it is very helpful to analyze the distribution plots for hexagonally ordered adlayers presented in Figs. 1 and 3 . The set of $(\sqrt{7} \times \sqrt{7}) R 19.1^{\circ}$ structures is limited to one with threefold and atop adsorption sites, marked as (g) (with minimum average adlayer height and maximum roughness), and another one with registry between atop and bridge sites, marked as (h) (with maximum average adlayer height and minimum roughness). Indeed, we believe that the $(\sqrt{7} \times \sqrt{7}) R 19.1^{\circ}$ adlayer formed by $\mathrm{Xe}$ on $\mathrm{Pd}(111)$ 
corresponds to structure (h). This is due to a tendency of Xe to occupy an atop site. This conjecture is further supported by the fact that an intermediate maximum between structures (k) and (h) on Fig. 3 is the $(\sqrt{19} \times \sqrt{19}) R 23.4^{\circ}$ structure with $\theta=0.37$, which was also reported for Xe-Pd(111). ${ }^{18}$ Furthermore, the $(\sqrt{7} \times \sqrt{7}) R 10.9^{\circ}$-symmetry structure was reported for $\mathrm{Ar}-\mathrm{Ag}(111)$ system by Caragiu et $a .^{19}$ and $(3 \times 3)$-symmetry structure was found in the case of $\mathrm{Kr}-$ $\mathrm{Ru}(0001)$ by Narloch and Menzel. ${ }^{20}$

\section{CONCLUSIONS}

It is very encouraging to see how our rather simple model can be easily applied, with a great precision, for adsorbate-substrate systems with different interactions (chemisorption versus physisorption). Therefore, we believe that the approach presented here will facilitate the understanding of atomic adlayer formation, i.e., differences between halogen (or halide) and rare-gas adsorption on metal surfaces. To our knowledge, no such comparison between the halogen and rare-gas adlayer structures has been made to date. As suggested in the literature, in the case of the rare-gas adsorption, structures with occupancy of atop site (with maximum adlayer height in our study) are preferred. On the other side, the halogen hexagonal adlayers with minimum average adlayer height are favored. Our methodology based on evaluation of the average adlayer height and the adlayer roughness is very useful for understanding the adlayer characteristics and clearly demonstrates the structural differences due to the atomic registry. The parameters used in our model are clearly the most simple for the purpose of identification of the most likely structures. Although we did not test our approach on systems other than halogens and rare-gases adsorbed on metal surfaces, we have preliminary results showing that other systems could be described. We have recently shown that the I-Au(111) system, which possesses structures with long periodicity, could also be described by our unequal-sphere packing model. ${ }^{21}$ Moreover, additional pa- rameters could be used for identification of the most likely structures in the case of different adsorbate-substrate systems.

\section{ACKNOWLEDGMENTS}

The authors wish to gratefully acknowledge support by CONACYT and Laboratorio de Supercomputo y Visualizacion en Paralelo at UAM-Iztapalapa for access to its computer facilities.

${ }^{1}$ G. A. Somorjai, Introduction to Surface Chemistry and Catalysis (Wiley, New York, 1994).

${ }^{2}$ O. M. Magnussen, Chem. Rev. (Washington, D.C.) 102, 679 (2002).

${ }^{3}$ A. T. Hubbard, Chem. Rev. (Washington, D.C.) 88, 636 (1988).

${ }^{4}$ K. Horn and M. Scheffler, Handbook of Surface Science: Electronic Structure (North-Holland, Amsterdam/Elsevier, Amsterdam, 2000).

${ }^{5}$ R. I. Masel, Principles of Adsorption and Reaction on Solid Surfaces (Wiley, New York, 1996).

${ }^{6}$ R. Plass, J. A. Last, N. C. Bartelt, and G. L. Kellogg, Nature (London) 412, 875 (2001).

${ }^{7}$ R. Bennewitz, J. N. Crain, A. Kirakosian, J.-L. Lin, J. L. McChesney, D. Y. Petrovykh, and F. J. Himpsel, Nanotechnology 13, 499 (2002).

${ }^{8}$ A. Tkatchenko and N. Batina, Phys. Rev. B 70, 195403 (2004).

${ }^{9}$ A. Tkatchenko and N. Batina, J. Chem. Phys. 122, 094705 (2005).

${ }^{10} \mathrm{~S}$. Torquato, T. M. Truskett, and P. G. Debenedetti, Phys. Rev. Lett. 84, 2064 (2000)

${ }^{11}$ A. Donev, I. Cisse, D. Sachs, E. A. Variano, F. H. Stillinger, R. Connelly, S. Torquato, and P. M. Chaikin, Science 303, 990 (2004).

${ }^{12}$ A. Donev, F. H. Stillinger, P. M. Chaikin, and S. Torquato, Phys. Rev. Lett. 92, 255506 (2004).

${ }^{13}$ R. D. Diehl, T. Seyller, M. Caragiu, G. S. Leatherman, N. Ferralis, K. Pussi, P. Kaukasoina, and M. Lindroos, J. Phys.: Condens. Matter 16, 2839 (2004)

${ }^{14}$ C. A. Lucas, N. M. Marković, and P. N. Ross, Phys. Rev. B 55, 7964 (1997).

${ }^{15}$ J. L. F. D. Silva, C. Stampfl, and M. Scheffler, Phys. Rev. Lett. 90, 066104 (2003).

${ }^{16}$ J. F. Zhu, H. Ellmer, H. Malissa, T. Brandstetter, D. Semrad, and P. Zeppenfeld, Phys. Rev. B 68, 045406 (2003).

${ }^{17}$ B. Vogt, B. Kessler, N. Müller, G. Schönhense, B. Schmiedeskamp, and U. Heinzmann, Phys. Rev. Lett. 67, 1318 (1991).

${ }^{18}$ G. Hilgers, M. Pothoff, N. Müller, and U. Heinzmann, Surf. Sci. 322, 207 (1995).

${ }^{19}$ M. Caragiu, G. S. Leatherman, T. Seyler, and R. D. Diehl, Surf. Sci. 475, 89 (2001)

${ }^{20}$ B. Narloch and D. Menzel, Surf. Sci. 412, 562 (1998).

${ }^{21}$ A. Tkatchenko and N. Batina, J. Phys. Chem. B 109, 21710 (2005). 